\title{
Design and construction of a refracting telescope
}

\author{
C. I. Onah" ${ }^{*}$, C. M. Ogudo \\ Department of Physics, Federal University of Technology, Owerri, Nigeria
}

\section{Email address:}

cossyonah@gmail.co (C. I. Onah)

\section{To cite this article:}

C. I. Onah, C. M. Ogudo. Design and Construction of a Refracting Telescope. International Journal of Astrophysics and Space Science. Vol. 2, No. 4, 2014, pp. 56-65. doi: 10.11648/j.ijass.20140204.11

\begin{abstract}
Most people see the telescope as the things for the movies, the science geeks and the rich and affluent, but are these feelings for real? This paper on the design and construction of an optical refracting telescope which is aimed at producing a low cost and portable telescope with less or no aberration effects using the materials we see around us every day goes a long way to answer the question that the telescope is for everybody that loves astronomy. Overall implementation of this work involves knowledge of the physics of optics; lenses to be precise. As a case study I used a double convex lens and the eyepiece of a microscope for the construction of the mini refractor telescope, my hypothesis is that using a double convex is better than using a Plano-convex because the two curved surfaces will cancel out the aberration effect of the individual sides. The resultant telescope was tested during the night and during the day and was used to focus objects at a distance of about $50 \mathrm{~m}$ from the person with less aberration effect.
\end{abstract}

Keywords: Telescope, Astronomy and General Physics

\section{Introduction}

Telescope can be defined (e.g. King, 1955; Smith \& Thompson 1988) as an instrument that increases the angular separations of a range of plane waves and improves the limit of the eye's resolution. Some researchers (e.g. Rene Descartes 1637) in the words of a philosopher described the telescope as a wonderful instrument that open the way to a deeper and more perfect understanding of nature. Oxford Dictionary of Science defined the telescope as an instrument used to collect radiation from a distant object in order to produce an image of the object or enable the collected radiation to be analyzed. However, from our own perspective, a telescope is an instrument used to view distant objects clearly and apparently magnified by collecting electromagnetic radiations. When an object is viewed through a telescope it appears closer and therefore larger, thus the telescope increases the visual but not the size of an object one is looking at (that is; it does not make an object bigger than it already is). Suppose one is looking at the moon through a telescope the image of the moon will appear bigger because the angle it subtends at the eye is larger but when the same moon is viewed with the naked eye, the visual angle is small and so the moon appears to be small. The telescope is different from a microscope in that a telescope is used to view distant object while the microscope is used to view objects close at hand, also the focal length of the objective lens in a telescope is usually longer than that of the eyepiece while the reverse is the case in a microscope (i.e. the focal length of the eyepiece is longer than that of the objective).

\subsection{Theory of Telescopes}

There is some documentary evidence, but no surviving designs or physical evidence, that the principles of telescopes were known in the late 16th century. Writings have ascribed the use of both reflecting and refracting telescopes to Leonard Digges, and was independently confirmed by a report by William Bourne in approximately 1580. They may have been experimental devices and were never widely reported or reproduced. Although Digges may have created a rudimentary instrument involving lenses and mirrors but the optical performance required to see the details of coins lying about in fields, or private activities seven miles away, was far beyond the technology of the time.

The documented and proven credit of the origin of telescope has been attributed to three individuals; Hans Lippershey, Sacharias Jansen and Jacob Metius. And their design was widely known as the Dutch telescope. But it 
wasn't until 1610 when Galileo demonstrated his design of a telescope which was an improved work of the Han Lippershey telescope constructed in 1608 that the instrument was given the name "telescope" and so the seventeenth (17th) century saw the creation of the optical telescopes which is the most popular of all telescope types and the foundation for all other telescope designs. Galileo's design is the known as the refracting telescope. Other types of the optical telescope that was invented in the $17^{\text {th }}$ century includes; Johannes Kepler's variation of the Galilean telescope in 1611 and sir Isaac Newton's refracting telescope which used curved mirrors instead of lenses in 1704.

The $20^{\text {th }}$ century saw the evolvement of telescopes of other wavelength like the infrared, radio, ultraviolet, gamma telescope etc. and the era of adaptive optics like the advanced optics system which was created due to man's desire to see more than the normal eyes can. (King 1955)

\subsection{The Electromagnetic Spectrum}

Electromagnetic waves are produced by accelerating charges which transport energy and momentum from source to a receiver. The electromagnetic waves travel in a vacuum at the speed of light and are of different types which are only distinguishable by their wavelength and frequencies. The list of the different types of electromagnetic waves and their wavelength and frequencies is called the electromagnetic spectrum.

Newton based on his theory of light argued that light is made of particles and not waves and that these particles combine to appear white, thus introducing the concept of coloured spectrum. So light of different wavelength and frequencies are represented in the electromagnetic spectrum in terms of colour.

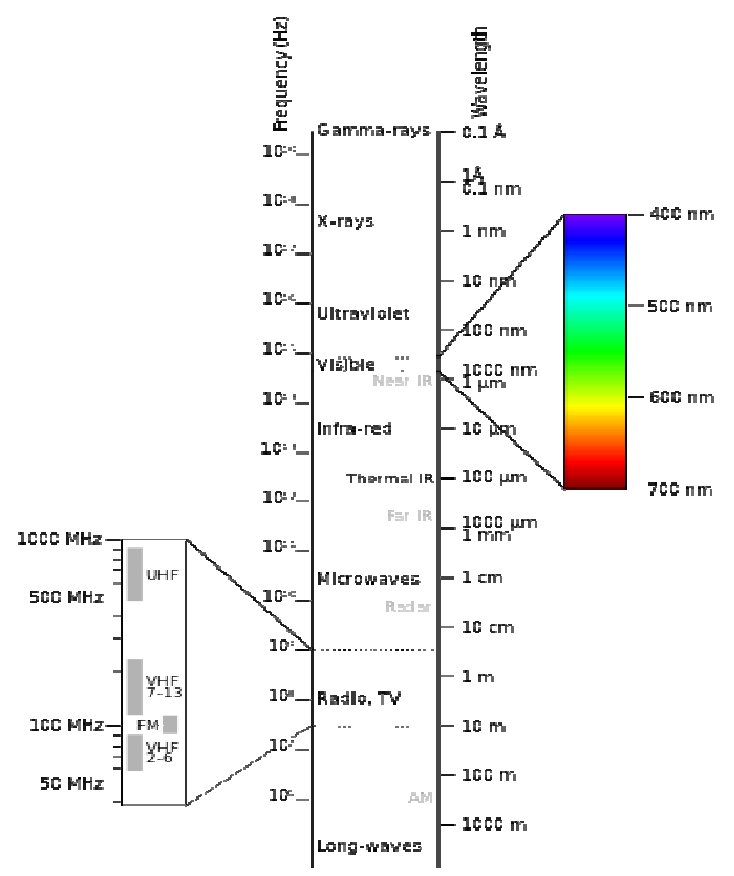

Fig 1.1. The electromagnetic spectrums (Keiner, 2011)

\subsection{Optical Telescopes and Types}

Optical telescope is the most common of all telescope types and it is a telescope that focuses and gathers light mainly from the visible part of the electromagnetic spectrum creating a magnified image for direct view. The optical telescope uses optical components like mirrors, lenses and solid state detectors to observe light from the near infrared to the near ultra-violet wavelengths. From the electromagnetic spectrum, wavelengths of approximately $400 \mathrm{~nm}$ (purple) to $700 \mathrm{~nm}$ (red) all fall under the visible part of the spectrum and can be detected with the eyes. There are three main types of optical telescopes (subrahmanyam, et al. 2007). These are refracting, reflecting and catadioptric telescopes.

\subsection{The Refracting Telescope}

A refracting telescope is an optical telescope that uses lens as its objective to form image. It got its name from the fact that its image is formed by bending of light or refraction. A refractor can be a combination of a convex lens and a concave lens or a combination of two convex lenses. It is also known as dioptrics (smith \& Thompson. 1988). It is made up of the objective or primary lens which is used to refract or even bend the light so that it converges at a focus point; this lens is usually thicker at the center than it is toward its edges, which bends the light more at the edge of the lens than light coming through the centre. This allows all of the light to come together at a focus point. The point of focus is where the image is created and the eyepiece (a concave lens) helps the human eye in gathering light and magnifies the image. These two lenses are fixed at the ends of two coaxial tubes and parallel rays from an astronomical or distant object enters the telescope through the objective lens and the image formed is real, inverted and diminished at the focal point of the objective lens. If the image formed is adjusted to be at the focal point of the eyepiece, the final image will be formed at infinity and the image is therefore highly magnified and erect. This is known as the normal adjustment of a telescope.

There are two main types of refractors: the achromatic refractors which uses an achromatic lens (a lens made of two pieces of glass with different dispersion), a typical example of this is the Great Paris Exhibition Telescope of 1990 and the Apo-chromatic refractors which have objectives built with extra-low dispersion materials and designed to bring three different wavelengths (red, green, blue) into focus in the same plane because the objective is made of three pieces of glass. (Hetcht, 1987).

This work will be focusing on refracting telescope (dioptrics) mainly because it is the foundation for other telescope designs, in that when its concept is understood properly, one can develop on that to design other telescope types and because of the availability of the materials for it than that of others. 


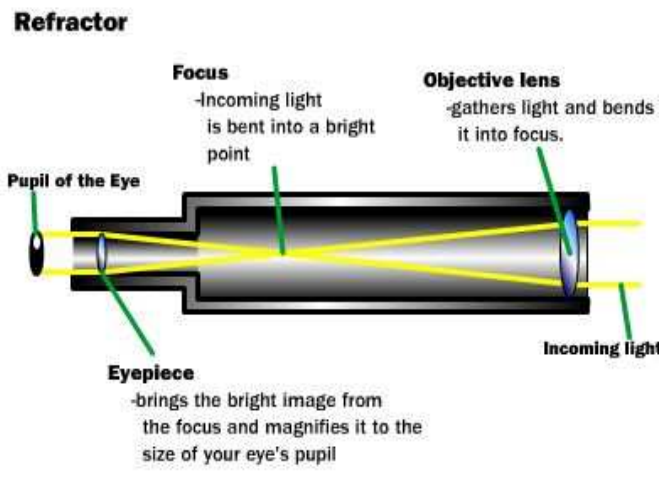

Fig. 1.2. A typical structure and working of a refractive telescope (howstuffwork, 2000).

\subsection{Principles of Refraction}

The law of refraction predicts that a light ray always deviates towards the normal in the optically dense medium and so the direction of light is changed at the boundary of glass and air by refraction. By designing lenses having the right curvature, this principle can be used to gather and focus light. On these laws, the type of refraction and image formation for a converging lens can be summarized under these three principles:

- Any incident ray travelling parallel to the principal axis of a converging lens will refract through the lens and travel through a focal point on the opposite side of the lens

- Any incident ray travelling through the focal point on the way to the lens will refract through the lens and travel parallel to the principal axis.

- An incident ray travelling through the center of the lens will continue in the same direction that it had when it entered the lens.

\subsection{The Reflecting Telescopes}

This is a type of an optical telescope that uses a single or a combination of curved mirrors instead of lenses to focus or reflect light and produce images. This was invented in the $17^{\text {th }}$ century as an alternative to the refracting telescope which at that time suffered from chromatic aberration. Although the reflector also suffers from other forms of aberration it allows for very large diameter of objectives and so widely used in astronomy. A typical reflector reflects light from a concave mirror which is the objective; the reflected light is reflected again by a plane mirror placed $45^{\circ}$ in a way that the reflected light will pass through the eyepiece to the observer. This is known as the Newtonian telescope, other type of reflectors developed from this design are; the Gregorian reflector by James Gregory, the Cassegrain reflectors by N. Cassegrain and others which uses different types of secondary optical systems. Reflectors are also known as catoptrics (Hetcht, 1987).

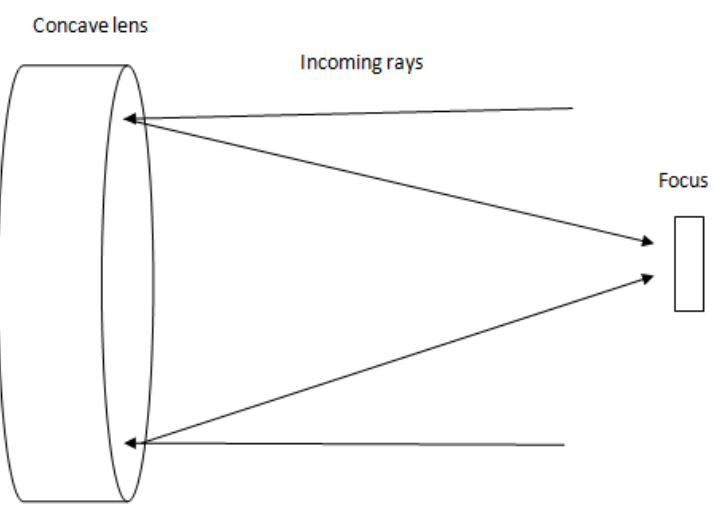

Fig. 1.3. A typical reflector

\subsection{Catadioptric Telescope}

A catadioptric optical system uses a combination of refraction and reflection usually by using lenses (dioptrics) and curved mirrors (catoptrics). This is usually done so that the telescope can have an overall greater degree of error corrections than the refracting and reflecting telescopes. They are easier to manufacture and many of them employ correctors, a lens or a curved mirror in a combined imageforming optical system so that the reflective or refractive element can correct the aberrations produced by its counterpart. The first of this type of telescope was the Hamiltonian telescope by W. F. Hamilton in 1814 and the schupmann medial telescope by Ludwig Schulman near the end of the $19^{\text {th }}$ century other examples include the SchmidtCassegrrain telescope, Maksutov-Cassegrain telescope, Argunov-Cassegrain telescope etc. (Hetcht, 1987).

\subsection{Characteristics of a Refracting Telescope}

\subsubsection{Focal Length and Focal Ratio}

The focal length of an optical system is a measure of how strongly the system converges or diverges light. For an optical system in air, it is the distance over which initially collimated rays are brought to a focus and in simple English is the distance from the centre of the lens to the point where light rays passing through it converges. A system with a shorter focal length has greater optical power than one with a long focal length that is; it bends the rays more strongly, bringing them to a focus in a shorter distance.

Mathematically the focal length $\mathrm{f}$ is given by

$$
\frac{1}{\mathrm{f}}=\frac{1}{\mathrm{~d}}+\frac{1}{\mathrm{D}}
$$

The focal point is also half of the center of curvature of the lens i.e.

$$
f=\frac{c}{2}
$$

In astronomy, the f-number is commonly referred to as the focal ratio of a telescope and is defined as the focal length $\mathrm{f}$ of an objective divided by its diameter $\boldsymbol{V}$ or by the diameter of an aperture stop in the system. The focal length 
controls the field of view of the instrument and the scale of the image that is presented at the focal plane to an eyepiece, film plate, or CCD. This is written as "f/" and then the calculated value which is often given along with the diameter of the objective to describe the scope. For example this work is the construction of $50 \mathrm{~mm}$ " $\mathrm{f} / 4$ " refractors

\subsubsection{Magnification}

The magnification through a telescope magnifies a viewing object while limiting the field of view (FOV). This can be expressed as:

$$
\mathrm{M}=\frac{\mathrm{F}}{\mathrm{f}}
$$

where $F$ is the focal length of the objective lens, and $f$ is the focal length of the eyepiece. Magnification is often mistaking as the optical power of the telescope, its characteristic is the most misunderstood term used to describe the observable world. At higher magnifications the image quality significantly reduces. Similar minor effects may be present when using star diagonals, as light travels through a multitude of lenses that increase or decrease effective focal length. The quality of the image generally depends on the quality of the optics (lenses) and viewing conditions - not on magnification. If the magnification of the telescope is positive, the image produced will be upright otherwise it will be inverted for a negative value of magnification.

Magnification itself is limited by optical characteristics. With any telescope or microscope, beyond a practical maximum magnification, the image looks bigger but shows no more detail. It occurs when the finest detail the instrument can resolve is magnified to match the finest detail the eye can see. Magnification beyond this maximum is sometimes called empty magnification. To get the most detail out of a telescope, it is critical to choose the right magnification for the object being observed. Some objects appear best at low power, some at high power, and many at a moderate magnification. There are two values for magnification, a minimum and maximum. A wider FOV eyepiece may be used to keep the same eyepiece focal length whilst providing the same magnification through the telescope. For a good quality telescope operating in good atmospheric conditions, the maximum usable magnification is limited by diffraction.

\subsection{The Resolution of an Optical Telescope}

Apart from magnification of an image, other important functions of the telescope are its resolving power and its light gathering power. Resolving power or Resolution is the ability of the telescope to distinguish between objects. And resolving power is a measure of how well you can separate two objects, and of course this is related to how sharp the image looks. Increasing the resolution allows more detail of the image to be seen or for the image to be clearer. Under favorable condition, the largest optical telescope can attain a resolution of better than one sec of arc, good enough to distinguish between a pair of car headlamp at a distance of about $300 \mathrm{~km}$. Also most people think that the function of a telescope is to make things bigger but the actual fact is that it makes things brighter and this is called its "light gathering power". Both of these functions; light gathering power and resolving power depends only on the size of the telescope called APERTURE. The light gathering power depends on the area of the objective while the resolving power depends on the diameter of the objective. A telescope of aperture 2D has four times the light gathering power and twice the resolving power of a telescope of aperture D. Also the resolving power depends inversely on wavelength while light gathering power does not depend on wavelength and thus the relationship between resolving power and aperture size is:

Angular resolution, $(\mathrm{R})$ in $(\operatorname{arcsec})=0.25 \mathrm{X}$ wavelength (microns) / telescope diameter $(\mathrm{m})$ i.e.

$$
\mathrm{R}=0.25 \frac{\lambda}{\mathrm{D}}
$$

\subsection{Field of View}

Field of view (FOV) is the extent of the observable world seen at any given moment, through an instrument (e.g., telescope or binoculars), or by naked eye. This is dependent on the specifications of the eyepiece or on both that of the eyepiece and the telescope. There are different types of field of view. Apparent FOV is the observable world observed through an ocular eyepiece without insertion into a telescope. A wider FOV may be used to achieve a vaster observable world given the same magnification compared with a smaller FOV without compromise to magnification. Note that increasing the FOV lowers surface brightness of an observed object as the gathered light is spread over more area; in relative terms increasing the observing area proportionally lowers surface brightness dimming the observed object. Wide FOV eyepieces work best at low magnifications with large apertures, where the relative size of an object is viewed at higher comparative standards with minimal magnification giving an overall brighter image to begin with.

True FOV is the observable world observed though an ocular eyepiece inserted into a telescope. Knowing the true FOV of eyepieces is very useful since it can be used to compare what is seen through the eyepiece to printed or computerized star charts that help identify what is observed. True FOV, $v_{t}$, is expressed as:

$$
v_{t}=\frac{v_{a}}{M}
$$

where, $v_{t}$ is the true field of view $v_{a}$ is the apparent field of view and $\mathrm{M}$ is magnification of the telescope. Maximum FOV is a term used to describe the maximum useful true FOV limited by the optics of the telescope. 


\subsection{Limitations of the Refracting Telescope}

The refracting telescope has so many defects ranging from aberration effects, lens sag for larger diameter of lenses but I would briefly discourse the aberration effect because it directly affect this present work.

\subsection{Aberration Effect}

Aberration is the departure of real images from the ideal images in respect to the actual size, shape and position. In other words it is any failure of a lens or mirror to behave precisely as it is supposed to. There are two types of aberration effect; the mono-chromatic aberration which includes spherical aberration, coma and astigmatism which deteriorate the image thereby making it unclear and distorting the field curvature which deforms the image.

\subsection{Chromatic Aberration}

Since refraction varies with wavelength and a single lens has different powers for different colors, when light which is composed of radiation of many colours passes through a lens, different colours are diffracted by different amounts. Blue light comes to a focus closure to the lens than red light and the image formed will appear to be blue on one side and orange on the other side. There are two types of chromatic aberration; the longitudinal and lateral chromatic aberration.

\subsection{The Longitudinal Chromatic Aberration}

This occurs when different wavelengths of light are focused at different distances from the lens i.e. when the objective lens cannot focus different wavelength at the same focus longitudinal chromatic aberration occurs. It is caused by straight incident light. The foci of the different colours lie at different points in the longitudinal direction along the optical axis. This leads to coloured areas in the images that arise because not all three colours can be displayed

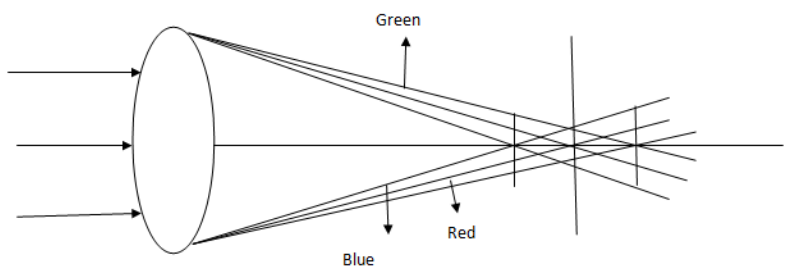

Fig. 1.4. The longitudinal chromatic aberration

\subsection{The Lateral Chromatic Aberration}

When an eyepiece focal length varies with wavelength, and magnification is inversely proportional to the eyepiece focal length, the magnification will be different at different wavelength, this leads to lateral chromatic aberration. This kind of aberration does not lead to coloured areas but to fringes that occur around objects of high contrast.

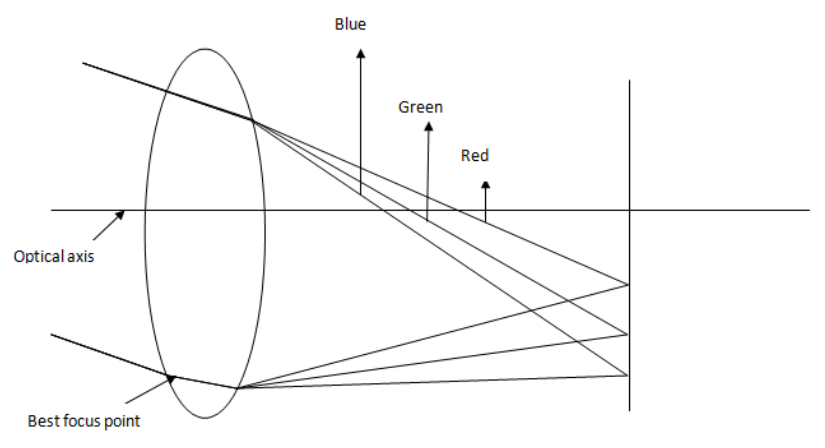

Fig 1.5. Lateral chromatic aberration

\subsection{Correction of Chromatic Aberration}

To correct chromatic aberration, the best known method is to use two lenses in contact of different intensities that are made of crown and the flint respectively. The former has a moderate dispersion while the later has a high dispersion. When combined, the two dispersions cancel each order making the image formed by the lens free of chromatic aberration. The other method used in correcting chromatic aberration is using the spaced doublet. A convex lens refracts beams of short wavelengths (blue) stronger than those of other wavelength (red) and the concave lens does the opposite of this when the two are used together, the different light beams meet in one focus.

\subsection{Importance of the Telescope}

The optical instrument, telescope is synonymous with astronomy or astrophysics and since most of the universe is invisible to us because we only see so much with the human eyes, the telescope is an important instrument in observatory astronomy and also helps give a better understanding of the universe. It is also useful in the fields of ornithology, pilotage and reconnaissance. It can also be used for watching sports or performance art.

This work will be a hallmark or the beginning of astrophysics for one and will create a deeper understanding of the physics of optics. This present work is to design and construct a reliable, maintainable and portable optical telescope that will be used in studying the nature and size of image formed when a convex lens is combined with an eyepiece. The structure and workings of a telescope together with the demonstration of the aberration effects and its correction will be studied.

\subsection{Advantages of the Refracting Telescope}

- The refractor telescope is generally lighter and smaller in optical tube assembly per inch of aperture.

- It is better for observing the moon and the planets.

- It is one of the rugged and long -lasting telescope designs.

- There is no telescope mirrors to adjust (no collimation need) or maintain due to the closed optical tube design. 
- It can also be used in daytime as well as at night.

\subsection{Disadvantages of the Refractor}

- It is most expensive per inch of aperture than other telescope designs.

- The objective lens can only be supported at the ends and so prone to sag under its own weight.

- How well light passes through decreases as the thickness of the lens increases.

- It is difficult to make a lens with no imperfections inside the lens and with a perfect curvature on both sides of the lens.

- Suffers from chromatic aberration.

- How well light passes through the lens varies with the wavelength of the light. Ultraviolet light does not pass through the lens at all and so cannot be used for deep-sky objects and bodies.

The first known refracting telescope was Galileo's design of the refractor in 1609 using a convex lens as the objective and a concave lens as the eyepiece. This design has no intermediary focus and so the image produced was non-inverted and upright. But the major fault of this telescope although used to view the satellites of Jupiter suffered from chromatic aberration.

Johannes Kepler (1611) suggested a variation in the Galileo's design of the refractor and so used a convex lens as an objective and a convex lens as an eyepiece too. The advantage of his design over Galileo's is that the light emerging from the eyepiece are converging and so allows for a much wider field of view and a greater eye relief but the image is inverted. Also just like Galileo's design this one also suffered from chromatic aberration and produced blurry image. (King, 1955)

Over 400 years has passed since the time of the invention of the first refracting telescopes and it was generally supposed that chromatic errors seen in lenses simply arose from errors in the spherical figure of their surfaces. Opticians tried to construct lenses of varying forms of curvature to correct these errors.

Isaac Newton discovered in 1666 that chromatic colours actually arose from the uneven refraction of light as it passed through the glass medium. This led opticians to experiment with lenses constructed of more than one type of glass in an attempt to cancelling the errors produced by each type of glass. It was hoped that this would create an "achromatic lens"; a lens that would focus all colours to a single point, and produce instruments of much shorter focal length. The lens they then created as the objective of a refractor is a convex lens coated with flint and crown to cancel out aberration effect. (King, 1955)

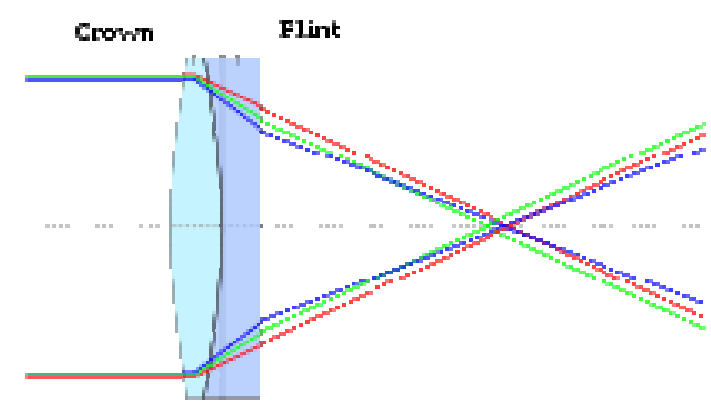

Fig. 2.1. Light rays through an achromatic lens (King, 1955)

Since after the designs of both Galileo and kepler, many scientist and artist has continued to develop on their work, creating new designs and with different methods and high power lenses in a bid to correct aberration effect. Some of these recent works from literature are discussed briefly below. Dickinson (2011) constructed a $16 \mathrm{~cm} \mathrm{f} / 5.9$ refractor with an objective lens made of crown and flint glass in a two-element sandwich design. It focuses sharply the visible spectrum but leaves some blue and red colours, in other words it suffered from chromatic aberration. Schroeder (2009) constructed a small table top refractor telescope for his $7 \mathrm{yr}$ old daughter using three different eyepieces, a Barlow lens as the objective and experimented using the three different eyepieces and was able to view the moon with two out of three of the eyepieces.

In Gary 2010, a 16cm f/5 refractor was constructed. The telescope's features include a short-focus achromatic objective lens from A. Jaegers Jr. Optics Inc., polyvinyl chloride (PVC) altitude bearings, a machined steel adapter, and a set of adjustable clamps to further control the altitude motion. The scope also has a plywood tripod that supports the rocker box and elevates the eyepiece to comfortable heights.

Dick (2005) in his mirror workshop constructed a 6inch $\mathrm{f} / 15$ refractor with objective lens made from readily available optical glass. It was used to view the stars with the green colour filtered out and the nearby planets without aberration effect.

Everyday new articles and suggestions on how to correct this defect in a refractor keeps pouring in and research are being carried out on this theories to verify and see if it will work and so high powered lenses that is a far cry from what Galileo and kepler used for their work are fabricated. In this present work, I suggest that a combination of two high powered lenses as the objective (a convex and concave) and the use of Ramsden or Huygens design of an eyepiece will take care of the problem of chromatic aberration

\section{Materials and Methodology}

\subsection{Design Objective}

In implementing this work, certain objectives were born in mind. These are outlined below 
- The object will be an optically excellent work that can be accomplished or built in a common workshop or the back of a family house.

- It should be able to see at least the craters of the moon.

- It should be portable (not too small or too big or long but just the right size).

\subsection{Design Specification}

- A hollow coaxial tube with both ends open.(diameter $50 \mathrm{~mm}$ )

- A $38 \mathrm{~mm}$ PVC pipe

- A $38 \mathrm{~mm}$ pipe adapter

- A convex lens. $(\mathrm{f} / \mathrm{l}=20 \mathrm{~cm})$

- An eyepiece $(\mathrm{f} / \mathrm{l}=16 \mathrm{~cm})$

\subsection{Block Diagram}

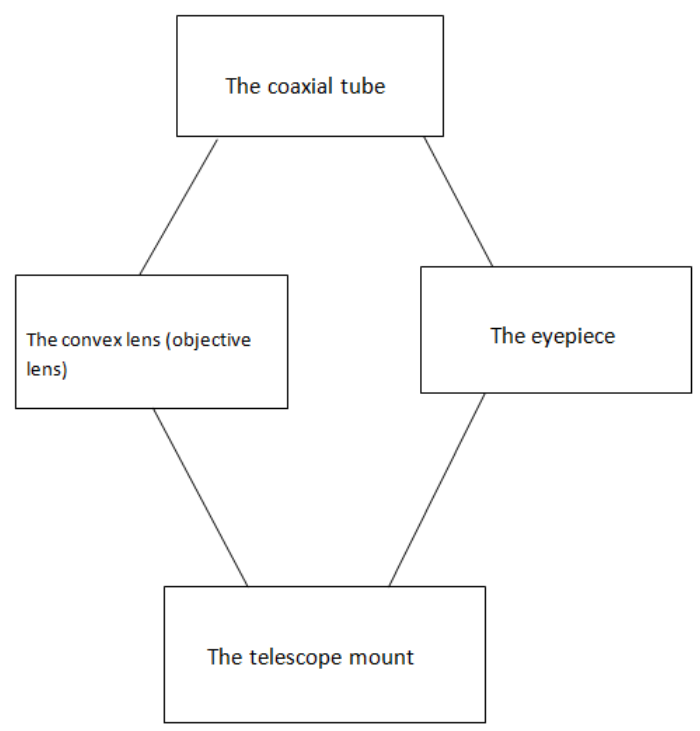

Fig. 3.1. Block diagram of a telescope

\subsection{Description of Materials Used and Theory of Operations}

\subsubsection{The Objective Lens}

The objective of an achromatic refracting telescope is an achromatic lens which is a convex lens and it is used to focus, refract or bend light that strikes on it. The refracted light then converges on a focus point.

The objective lens of a refractor comes in various diameters (aperture) and focal length, the most common is the $50 \mathrm{~mm} ; 20 \mathrm{~cm}$ convex lens and is limited to $107 \mathrm{~cm}$ so that the lens can be supported by its edges and if it is longer it will result in a distorted image. So the largest practical size of the objective lens of a refractor is 1 meter. In this design, we are using a $50 \mathrm{~mm} ; 20 \mathrm{~cm}$ convex lens as the objective lens of my telescope.

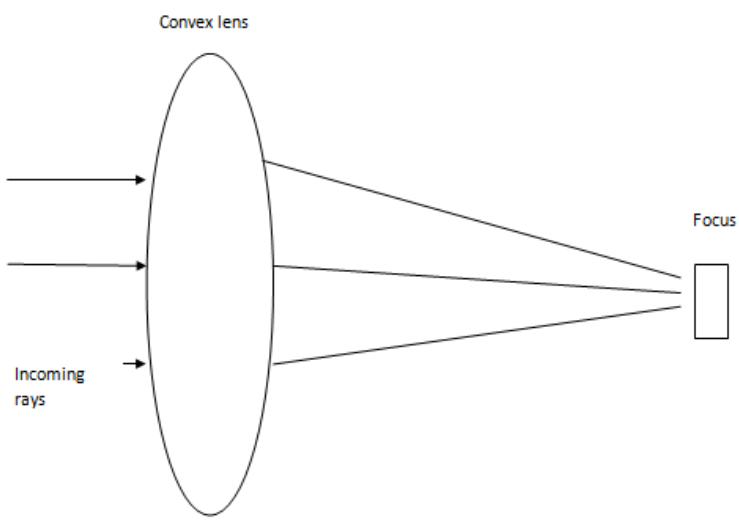

Fig. 3.2. A typical objective lens showing light rays

\subsubsection{The Eyepiece}

The eyepiece is the lens used to enlarge the image focused by the objective lens to form the final image which is then viewed or channeled to the eye. An eyepiece consists of multiple lenses used to reduce the work of the eye in focusing light. The focal length of an eyepiece is the distance from the principal axis of the eyepiece where parallel rays converge to a single point. When in use the focal point of the eyepiece combines with that of the objective lens to determine the magnification of the image one is viewing. This is often measured in millimeters. There are four types of eyepiece. These are Kellner's eyepiece which consists of two Plano-convex lens of equal focal length, used in microscopes and high power telescopes because of its large field of view.

Huygens eyepiece consists of two lenses of focal length in the ratio of $3: 1$ and placed at a distance equal to the difference in their focal length. It is free of chromatic aberrations because the distance between the lenses is equal to half of the sum of their focal length. Also spherical aberration is minimum but it has a small field of view. This is the eyepiece that will be used for this work. This is achieved by placing the curved side of the lenses facing each other

Others types of eyepiece include the Ramsden eyepiece consisting of two Plano-convex lenses each of focal length separated by a distance equal to $2 / 3$ of its focal length placed with their curved surfaces facing each other thereby reducing spherical aberration and free of chromatic aberration with a fairly wide field of view. Finally, we have the Gauss eyepiece which is a variation of the Ramsden eyepiece.

\subsubsection{The Coaxial Tube}

This is the tube that houses both the objective and the eyepiece of a telescope, for simplicity it can be made of wood, a carpet roll or a polyvinyl chloride (PVC) pipe or even a paper towel and cardboard paper of proper length and dimension can be used and is usually wrapped with aluminum foil for beautification. In the present work a PVC pipes of diameters $50 \mathrm{~mm}$ and $38 \mathrm{~mm}$ is preferred. 


\subsubsection{The Telescope Mount}

A telescope mount is a mechanical structure which supports a telescope. These are designed to support the mass of the telescope and allow it for accurate pointing of the instrument. Many sorts of mounts have been developed over the years, with the majority of effort being put into systems that can track the motion of the stars as the Earth rotates. We have the

Altazimuth, altitude-azimuth, or alt-az mounts which allows telescopes to be moved in altitude, up and down, or azimuth, side to side, as separate motions. This mechanically simple mount was used in early telescope designs but since the invention of digital tracking systems, altazimuth mounts have be used in all practically modern large research telescopes. Digital tracking has also made it a popular telescope mount used in amateur astronomy. Others include the alt-alt (altitude-altitude) mounts, the equatorial mounts (common with reflectors) and the hexapod telescope mounting system. But in the present work, it will be portably used without a telescope mount.

\section{Construction Procedure}

\section{Step One: Determining the Focal Length of the Lenses}

This can be done by writing large characters of letters on a plane sheet and holding each lens above it to determine its focal length till the image produced is bright, then measurement in centimeters is taken. This can also be done using a flashlight; in fact it is the best procedure to use when determining the focal length of any lens and can be achieved by using it on a plane sheet and adjusting the lens. The point where all the light rays converge is the focal length.

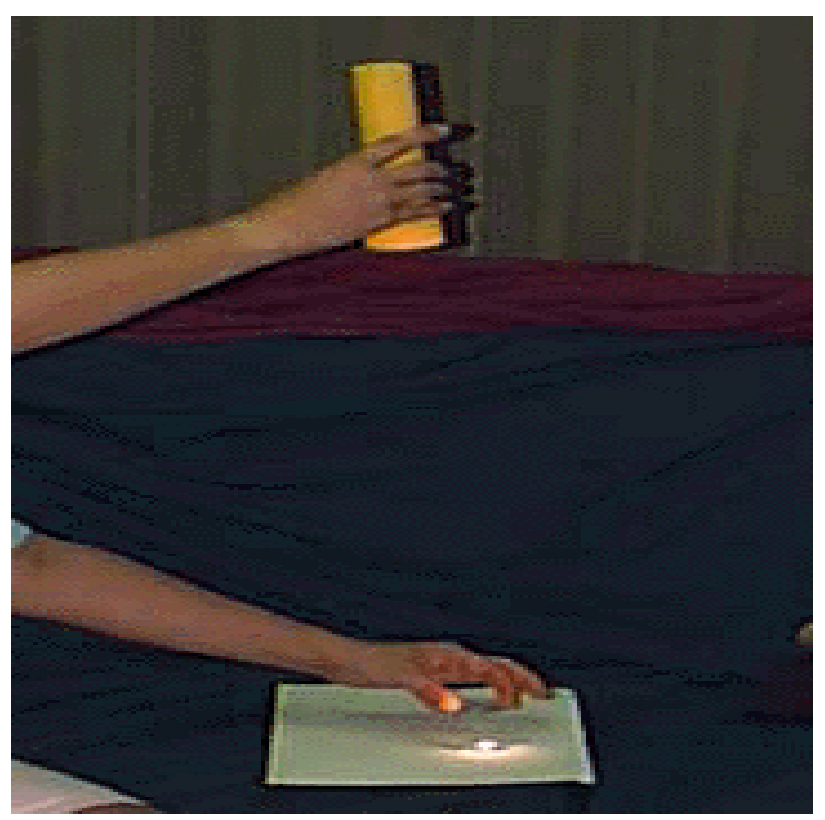

Fig. 3.3. Determining the focal length of the objective lens

\section{Step Two: Magnification of the Lenses}

The distance between the two lenses or the sum of their focal length results in the clearest magnification and in this case is $36 \mathrm{~cm}$. This can be determined by placing the eyepiece $7 \mathrm{~cm}$ from the eye and then slowly adjusting the objective until a clear image is formed and so using the equation for magnification, we have that;

$$
\mathrm{M}=\frac{\mathrm{F}}{\mathrm{f}}
$$

Where $\mathrm{F}$ is the focal length of the objective and $\mathrm{f}$ is the focal length of the eyepiece

$$
\mathrm{M}=\frac{20 \mathrm{~cm}}{16 \mathrm{~cm}}=1.25
$$

Thus the telescope will have 1.25 times magnification.

\section{Step Three: Building the Telescope}

Cutting the PVC pipe: the length of the $50 \mathrm{~mm}$ pipe will be the focal lengths of the objective $(20 \mathrm{~cm})$ with about $3 \mathrm{~cm}$ allowances, making it a total length of $23 \mathrm{~cm}$. The length of the $38 \mathrm{~mm}$ pipe will be the focal length of the eyepiece with the same amount of allowance. This is measured, cut out and painted black because black colour gathers light while others scatters it (blackbody radiation) and left to dry out for some days.

Assembling the telescope: A pipe adapter is pressed into the $50 \mathrm{~mm}$ PVC pipe and ensured that the adapter is coupled properly. You may need to push the couple against a table or the floor. The objective lens is placed through the opening of the pipe. The objective lens should rest inside the couple, against the rim of the adapter. The pipe is placed on the floor with the adapter side down. The lens is assured that it is resting flat against the adapter inside the pipe and gently shook to be confirmed. The eyepiece is placed into one end of the $38 \mathrm{~mm}$ pipe and secured with adhesives or pipe adapter of the proper size. Then one end of the PVC housing the eyepiece is pushed into the PVC housing the objective lens so that they are held fast and cannot come off when adjusted by placing a pipe adapter at the other end of the pipe housing the objective lens.

\section{Results, Test and Discussions}

\subsection{Observation Using the Telescope}

At the completion of the work, the outcome is a mini homemade refracting telescope shown in the figure 4.1 below. 


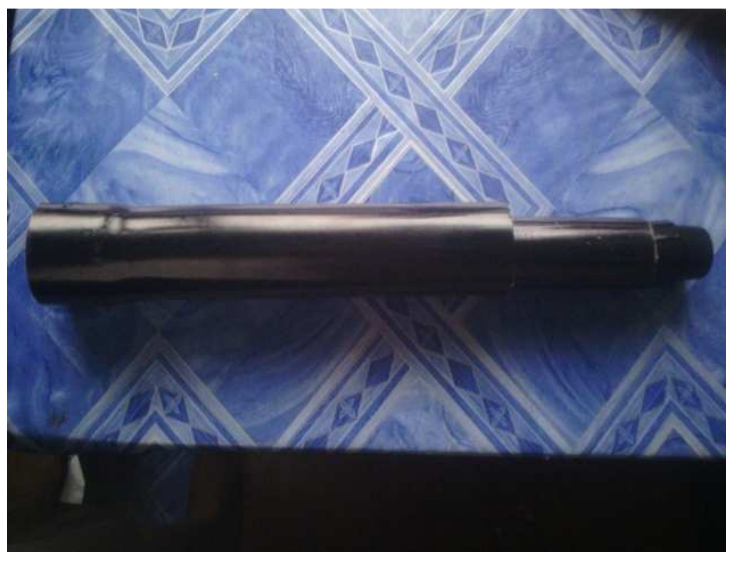

Fig. 4.1. The completed telescope

The most spectacular heavenly body to observe with a telescope is without a doubt the Moon. The best time to observe the Moon with a telescope is at the first quarter, when it appears only half illuminated. Under these conditions, lunar mountains and craters project long shadows, making them better visible from the Earth.

Encountering first observations with a simple telescope, the one with a small lens as objective such as the one in discussion here is recommended. The telescope is adjusted to its full length first, and then gradually adjusts until the object of focus is clear to the eye without distortion. Other objects observed are the nearest planets. Jupiter showed four satellites aligned along the equatorial plane, appearing as a model of the solar system. For observing the Rings of Saturn, one needs an instrument of good quality and high magnification power. The comparison between the apparent sizes of Jupiter and Saturn give you an idea of great distances in astronomy. We also observed Venus, which shows phases as the Moon, star clusters and double stars.

Unlike the Dickinson (2011) $16 \mathrm{~cm}$ f/5.9 refracting telescope which was used to view the craters of the moon and the nearby planets clearly, though with some aberration effect because of its high magnification and resolution which is a function of the diameter of the objective lens (the larger the diameter of the objective, the closer is the points it is able to distinguish as separated), the present one due to its small focal point and high resolution power viewed objects sharper with less aberration. During the day it can only focus near object of about $500 \mathrm{~m}$ without any aberration effect but the image formed is inverted which is a typical problem with refractors and during the night it can be used to focus objects farther with good light source but with some aberration effect.

\section{Summary and Conclusion}

Everyday people look up to the night sky some to imagine what the stars actually look like, others to the wonder of the different shapes that the moon take at different times of the year but only a few take this desire to know to a different level. Optical telescope form the basis for other telescopes in that for a radio telescope it still applies the principles used in constructing an optical telescope.

This telescope though small both in size and magnification was used to view object placed at a distance of about $500 \mathrm{~m}$ from it.

The essence of this work is to bring the telescope to us in a way that an amateur astronomer can take this desire to another level by using simple thing around us to construct the simplest telescope for astronomical observations. In science, it helps to understand the universe around us, bringing the terrestrial world to our doorstep and I hope that stars gazers like me will find this piece of work interesting and helpful.

Conclusively, to improve on this work, I recommend a lens of larger diameter and focal length than the one used in this work and also an improved eyepiece, possibly that of a camera to the eyepiece of a microscope for a better product.

\section{Acknowledgement}

We thank the anonymous referee for invaluable comments and suggestions that helped to improve the manuscript.

\section{References}

[1] Dick, P. (2005). www.mtbparker.com.Retrieved 15-02-2014

[2] Dickinson, T. (2011), SkyNews: the Canadian magazine of Astronomy \& stargazing, 17(2), 36.

[3] Elliot, R. S. (1966), Electromagnetism, McGraw-Hill Company Inc. New York. 138-153

[4] Gary, S. (2010). A 16cm; f/5 refractor, Sky and Telescope, 119(5), 70 .

[5] Gary, S. (2011). A 16cm f/6 refractor, Sky and Telescope; 121(5), 64

[6] Hetcht, E. (1987). Optics, Adelphi University, $2^{\text {nd }}$ Edition. Addison-Wesley Publishing Company, Canada. 174

[7] Journal on Refracting Telescope - a Closer look. (March 2013), Retrieved 05-06-2013

[8] Meyer-Arendt, J. R. (1972). Introduction to Classical and Modern Optics $3^{\text {rd }}$ Edition. Prentice-Hall Inc. New Jersey, USA. $122-126,159$

[9] Keiner, L.E., (2011) Coastal Carolina University @ www.unwittingvictims.com, the electromagnetic spectrum. Retrieved 16-02-2014

[10] King, H. C., ed. (1955). The History of Telescopes, Charles Griffin and co. Ltd, London, 74

[11] McGraw-Hill Dictionary of Scientific and Technical Terms, (2013). McGraw-Hill Company Inc., New York. 6E

[12] Mullins Mark, (1993), A Truly Economical Telescope, Sky and Telescope, 91-92 
[13] Oxford Dictionary of Science, $5^{\text {th }}$ Edition (2005). Oxford University Press Inc. New York, United States.

[14] Schroeder, P. (2009). In Defense of a Cheap Scope, Sky and Telescope, 118(3), 86
[15] Smith F. G., Thompson J. H., (1988), McGraw-Hill Company Inc. New York. $91-125$

[16] Subrahmanyam, N., Brij Lal, Avadhanulu, M.N. (2007). Textbook on Optics, S. Chand \& Company Ram Nagan, New Delhi. 173 